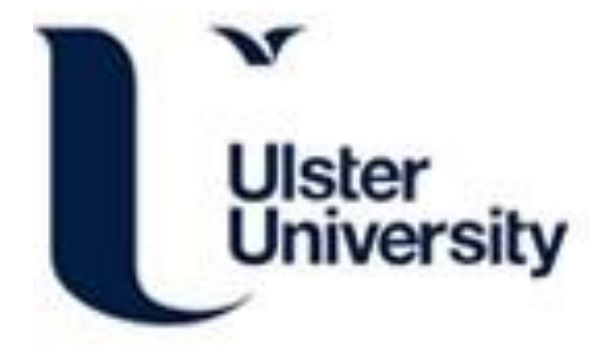

\title{
A two-fold classification for composite decision about localized arm movement from EEG by SVM and QDA techniques
}

Khasnobish, A., Bhattacharyya, S., Konar, A., Tibarewala, D. N., \& Nagar, A. K. (2011). A two-fold classification for composite decision about localized arm movement from EEG by SVM and QDA techniques. In 2011 International Joint Conference on Neural Networks, IJCNN 2011 - Final Program (pp. 1344-1351). [6033380] (Proceedings of the International Joint Conference on Neural Networks). https://doi.org/10.1109/IJCNN.2011.6033380

Link to publication record in Ulster University Research Portal

Published in:

2011 International Joint Conference on Neural Networks, IJCNN 2011 - Final Program

Publication Status:

Published (in print/issue): 24/10/2011

DOI:

10.1109/IJCNN.2011.6033380

Document Version

Author Accepted version

\section{General rights}

Copyright for the publications made accessible via Ulster University's Research Portal is retained by the author(s) and / or other copyright owners and it is a condition of accessing these publications that users recognise and abide by the legal requirements associated with these rights.

\section{Take down policy}

The Research Portal is Ulster University's institutional repository that provides access to Ulster's research outputs. Every effort has been made to ensure that content in the Research Portal does not infringe any person's rights, or applicable UK laws. If you discover content in the Research Portal that you believe breaches copyright or violates any law, please contact pure-support@ulster.ac.uk. 


\title{
A Two-fold classification for composite decision about localized arm movement from EEG by SVM and QDA techniques
}

\author{
Anwesha Khasnobish, Saugat Bhattacharyya, Amit Konar, D.N.Tibarewala and Atulya K. Nagar
}

\begin{abstract}
Disabled people now expect better quality of life with the development of brain computer interfaces (BCIs) and neuroprosthetics. EEG (electroencephalograph) based BCI research for robot arm control mainly concentrates on distinguishing the left/right arm movement. But for controlling artificial arm in real life scenario with greater degrees of freedom, it is essential to classify the left/right arm movement further into different joint movements. In this paper we have classified the raw EEG signal for left and right hand movement, followed by further classification of each hand movement into elbow, finger and shoulder movements. From the two electrodes of interest, namely, $\mathrm{C} 3$ and $\mathrm{C4}$, wavelet coefficients, power spectral density (PSD) estimates for the alpha and beta bands and their corresponding powers were selected as the features for this study. These features are further fed into the quadratic discriminant analysis (QDA), linear support vector machine (LSVM) and radial basis function kernelized support vector machine (RSVM) to classify into the intended classes. For left-right hand movement, the maximum classification accuracy of $87.50 \%$ is obtained using wavelet coefficient for RSVM classifier. For the multi-class classification, i.e., Finger-Elbow-Shoulder classification the maximum classification accuracy of $\mathbf{8 0 . 1 1 \%}$ for elbow, $\mathbf{9 3 . 2 6 \%}$ for finger and $81.12 \%$ for shoulder is obtained using the features obtained from power spectral density for RSVM classifier. The results presented in this paper indicates that elbow-fingershoulder movement can be successfully classified using the given set of features.
\end{abstract}

Keywords-EEG, BCI, wavelet transformation, PSD, QDA, LSVM, RSVM

\section{INTRODUCTION}

Rehabilitative aids have come a long way in the last decade. Starting with mere mechanical rehabilitative aids, disabled people are now expecting better quality of life with the development of brain computer interfaces (BCIs) and neuroprosthetics [1].

Anwesha Khasnobish, Saugat Bhattacharyya and D.N.Tibarewala are with the School of Bioscience and Engineering, Jadavpur University, Kolkata-700032, West Bengal, India. (phone: +91 99328 66197; ;email:anweshakhasno@gmail.com,saugatbhattacharyya@gmail. com, biomed.ju@gmail.com).

Amit Konar is with the Department of Electronics and Telecommunication, Jadavpur University, Kolkata-700032, West Bengal, India (email: konaramit@yahoo.co.in).

Atulya K. Nagar is with the Department of Math and Computer Science, Liverpool Hope University, UK. (email: nagara@hope.ac.uk)
Brain computer interfaces possess immense potential to bring the disabled people back to the mainstream of life providing means of communication bypassing the conventional neuro-muscular pathways.

Thus BCI based prosthetics can help people with devastating disorders like amyotrophic lateral sclerosis, brainstem stroke, cerebral palsy, spinal cord injury etc. [2] [3]. BCI translates the electrical signals produced in the brain due to the electrical activity, that are detectable on scalp or cortical surface or within the brain, into outputs that communicate the users' intent without participation of peripheral nerves and muscles[4].

BCI output can drive a word processor, speech synthesizer, cursor, robotic arm, wheel chair etc. Healthy users might communicate via BCI when conventional interfaces are inadequate, unavailable or too demanding [5-7]. A variety of brain activities monitoring methods provide the basis for brain computer interfaces. These include invasive and non-invasive methods like electrocorticography (ECoG), functional magnetic resonance imaging (fMRI), functional near infrared spectroscopy (fNIR), magnetoencephalography (MEG) and electroencephalography (EEG). Most research seen today in the BCI field is performed with EEG of its noninvasive technology to detect different characteristic signals emitted from brain. Though EEG has inferior spatial resolution, it provides better temporal resolution.

For motor control related BCI research the rolandic mu rhythm $(7-13 \mathrm{~Hz})$ and central beta rhythm (above 13 $\mathrm{Hz}$ ) originating from the sensory motor cortex are relevant [8]. During imagination or execution of body part movements, event related synchronization (ERS) in the gamma band and event related desynchronisation (ERD) in the mu and beta bands of the EEG originates in our brain. Contralateral to the movement, a decrease in mu and beta rhythm occurs with the movement or preparation of movement which is referred as ERD (event related desynchronisation). There occurs an increase in the power of mu and beta rhythms in the post movement phase, termed as ERS (event related synchronization) [9-14].

BCI system consists of modules to acquire brain signals, extract key features from them, and classify the features into intended classes ultimately aiming to translate into device commands. EEG based motor control study mainly relies on $\mathrm{C} 3, \mathrm{C} 4$ and $\mathrm{Cz}$, as these lie on the scalp above the motor cortex area associated with voluntary motor control [15]. Various features like the time domain and frequency domain parameters [1617], (STFT) [18], wavelet transforms, Spectral estimates 
[19], statistical parameters [20], Hjorth parameters [21], etc. are being used. Researchers have used various intelligent algorithms [22-30]viz. back propagation neural network, multilayer perceptron, discriminant analysis, support vector machines, vector quantization etc. to classify the EEG data in intended categories.

EEG based BCI research for robot arm control mainly concentrates on distinguishing the left/right arm movement. But for controlling artificial arm in real life scenario with greater degrees of freedom, it is essential to classify the left/right arm movement further into different joint movements. In this paper we have classified the raw EEG signal for left and right hand movement, followed by further classification of each hand movement into shoulder, elbow and finger movement (Fig.1), aiming the ability of the classifiers to make composite decision. These classifications will help in the realistic development of EEG based BCI control of artificial limb.

This paper is presented in six sections. An introduction to features extraction and classification is given in section II and III respectively. Section IV describes the complete experimental and signal processing procedures employed in this study. Performance analysis of extracted features and classifiers is given in section $\mathrm{V}$, followed by the conclusion in section VI.

\section{FEATURE EXTRACTION}

\section{A. Wavelet transformation}

Decomposing a signal into a set of basis functions are known as wavelets. These wavelets are obtained from a single prototype wavelet called the mother wavelet by dilations, contractions and shifting, which is the fundamental approach of wavelet transformation [19] [22] [31]. The mother wavelet function $\Psi_{a, b}(t)$ is given as

$$
\Psi_{a, b}(t)=1 / \sqrt{a} \Psi(t-b / a)
$$

where, $a, b \in R, a>0$, and $R$ is the wavelet space and ' $\mathrm{a}$ ' and ' $\mathrm{b}$ ' are the scaling factor and shifting factor respectively. The property of wavelet transformation to discriminate both temporal and spatial domain parameters make it an inevitable tool for feature extraction from EEG signals. The time frequency tradeoff encountered by short time Fourier transforms (STFT) is being overcome by wavelet transformations with their multi-scale approximation allowing effective localization of the signal with various spatio-temporal characteristics. Thus for a non-stationary signal like EEG, it is an effective analysis tool. The discrete wavelet transforms analyzes the signals at different resolutions by decomposing the signal into coarse approximation and detail information. Each level includes two digital filters and two down-samplers by 2 . The down-sampled outputs of the first high-pass and low-pass filters provide the detail D1 and approximation A1, respectively. The first approximation is further decomposed and the process is continued, until the desired level of decomposition is obtained. [32-33].

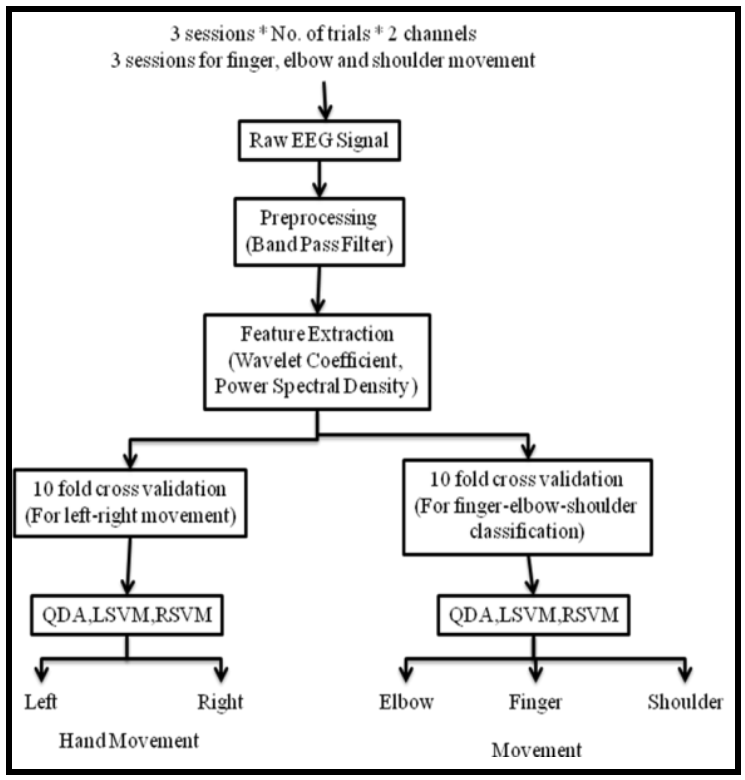

Fig.1. Proposed scheme

\section{B. Power spectral estimates}

Spectral density methods extract information from a signal to describe the distribution of its power in the frequency domain. The power spectral density (PSD) is defined as the Fourier transform (FT) of the signal's autocorrelation function, provided that the signal is stationary in a wide sense [33]. Thus for an EEG signal segmenting the complete time series data would be an ideal approach.

The measure for power spectral estimates is commonly divided into two methods; Non-parametric method and parametric method. The Welch's method fall into non-parametric method which, divides the times series data into overlapping segments, computing a modified periodogram of each segment and then the PSD estimates is averaged. Let $x_{m}(n)=x(n+m N)$, $n=0,1, \ldots N-1$, denote the $m$ th block of the signal $x \in$ $C^{M N}$, with $M$ denoting the number of blocks. Then the Welch PSD estimate is given by [34]

$$
R_{x}\left(w_{k}\right)=\sum_{m=0}^{M-1}\left|\operatorname{DFT}_{k}\left(x_{m}\right)\right|^{2}
$$

\section{ClASSIFIERS}

\section{A. Support Vector Machine}

Statistical learning theory being the basis of support vector machines (SVM) provides a new approach to pattern recognition. Support vector machines (SVMs) are a set of related supervised learning methods used for classification and regression [35-36]. They belong to a family of generalized linear classifiers. SVM training always finds global minimum and its performance depends upon the selected kernel, where the user chooses only the error penalty parameter. The foundations of Support Vector Machines (SVM) have been developed by Vapnik [37] and gained popularity due to many promising features such as better empirical performance. The formulation uses the Structural Risk Minimization (SRM) principle, which has been shown 
to be superior, [4], to traditional Empirical Risk Minimization (ERM) principle, used by conventional neural networks. SRM minimizes an upper bound on the expected risk, where as ERM minimizes the error on the training data. If the training data is labelled as $\left\{\boldsymbol{x}_{i}\right.$, $\left.y_{i}\right\}, i=1, \ldots, l, \quad y_{i} \in\{-1,1\}, \boldsymbol{x}_{i} \in \boldsymbol{R}^{d}$. Suppose there is some hyperplane which separates the positive from the negative examples (a "separating hyperplane"). The points $\mathbf{x}$ which lie on the hyperplane satisfy $\mathbf{w} \cdot \mathbf{x}+b=0$, where $\mathbf{w}$ is normal to the hyperplane, $|b| /\|w\|$ is the perpendicular distance from the hyperplane to the origin, and $\|w\|$ is the Euclidean norm of $\mathbf{w}$. Let $\mathbf{d}_{+}\left(\mathbf{d}_{-}\right)$ is the shortest distance from the separating hyperplane to the closest positive (negative) example. The "margin" of a separating hyperplane is defined as $\mathbf{d}_{+}+$ d_. The aim of linear support vector algorithm is to find the hyperplane with largest margin. Let us assume that all the training data satisfy the following constraints:

$$
\begin{aligned}
& \boldsymbol{x}_{i} \cdot \boldsymbol{w}+b \geq+1 \text { for } y_{i}=+1 \\
& \boldsymbol{x}_{i} \cdot \boldsymbol{w}+b \leq-1 \text { for } y_{i}=-1
\end{aligned}
$$

The above two equations can be combined to obtain the following resultant:

$$
\mathrm{y}_{\mathrm{i}}\left(\mathbf{x}_{\mathrm{i}} \cdot \mathbf{w}+\mathrm{b}\right)-1 \geq 0 \forall \mathrm{i}
$$

Considering the points for which the equality in (3) holds, these points lie on the hyperplane $\mathrm{H}_{1}: \mathbf{x}_{\mathrm{i}} \cdot \mathbf{w}+\mathrm{b}=$ 1 , where $\mathrm{w}$ is the normal and $|1-b| /\|w\|$ is the perpendicular distance from the origin. Similarly, the points for which the equality in Eq. (11) holds lie on the hyperplane $\mathrm{H}_{2}: \mathbf{x}_{\mathrm{i}} \cdot \mathbf{w}+\mathrm{b}=-1$. Hence $\mathrm{d}+=\mathrm{d}-=1 /\|w\|$ and the margin is $d_{+}+d_{-}=2 /\|w\|$.

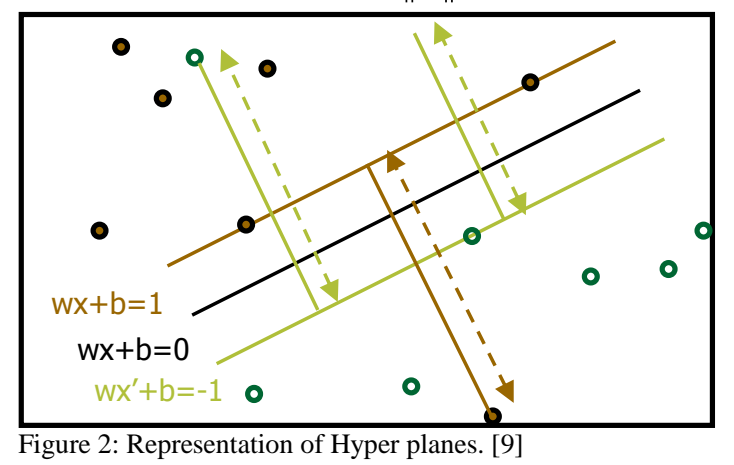

When the vectors are separated by non-linear region, the SVM uses a kernel function to map the data into a different space where a hyperplane can be used for separating the vectors. Certain function that corresponds to an inner product in some expanded feature space is referred to as kernel function. According to Mercer's theorem, every semi positive definite symmetric function is a kernel. Kernel function transforms the data into higher dimensional space to make it possible for the separation of the vectors (Fig. 13). The dot product becomes $K\left(\mathrm{x}_{\mathrm{i}}, \mathrm{x}_{\mathrm{j}}\right)=\varphi\left(\mathrm{x}_{\mathrm{i}}\right)^{\mathrm{T}} \varphi\left(\mathrm{x}_{\mathrm{j}}\right)$ when every data point is mapped into high-dimensional space via some transformation $\Phi: \quad \mathrm{x} \rightarrow \varphi(\mathrm{x})$. The kernel matrix, $\mathrm{K}_{\mathrm{ij}} \equiv \mathrm{K}\left(\mathrm{x}_{\mathrm{i}}, \mathrm{x}_{\mathrm{j}}\right)$, is a Gram matrix (a matrix of dot products (Horn, 1985)) in $\mathrm{H}$ (i.e. the Euclidean Space) [28]. It is necessary to choose $l$ training points such that the rank of the matrix $K_{i j}$ increases without limit as $l$ increases. The radial basis function is given by

$$
K\left(x^{t}, x\right)=\exp \left[-\frac{\left\|x^{t}-x\right\|^{2}}{\sigma^{2}}\right]
$$

which defines a spherical kernel where $x^{t}$ is the centre and $\sigma$, supplied by the user defines the radius.

\section{B. Quadratic discriminant analysis( $Q D A)$}

QDA is a generalized version of linear discriminant analysis (LDA), provided there are only two classes of points and the measurements are normally distributed. However unlike LDA, the assumption that the covariance of each class is identical is not taken into consideration in QDA [38-39]. Further, the surface that separates the subspaces will be a conic section (like parabola, hyperbola, etc.). The discriminant function is given by

$$
d_{k}(x)=2 \mu_{k}^{T} \sum_{k}^{-1} X-\mu_{k}^{T} \sum_{k}^{-1} \mu_{k}^{-2 \log \pi(k)}
$$

where, $\mathrm{k}=$ class, $\mathrm{X}$ is the set of measurements, $\boldsymbol{\mu}_{k}$ is the mean vector, $\pi_{k}$ is the prior probability and $\sum_{k}$ is the covariance matrix.

When (7) is multiplied by -2 , the discriminant function is given by

$$
d_{k}(X)=\left(X-\mu_{k}\right)^{T} \sum_{k}^{-1}\left(X-\mu_{k}\right)+\log \left|\sum_{k}\right|-2 \log \pi_{k}
$$

and the discriminant rule is given by

$$
d_{\hat{k}}(X)=\min _{1 \leq k \leq K} d_{k}(X) \Leftrightarrow \max _{1 \leq k \leq K} p(k / x)
$$

where, $p(k / x)=$ posterior distribution. Using this rule is called the QDA.

\section{DATA AND EXPERIMENTAL PROCEDURE}

The experiment is so designed to make the subject move their left-right hand along with moving a particular portion of the hand, namely, Finger, Elbow and Shoulder in a self paced manner.

\section{A. Subjects}

Five females and three males (right handed) in the age group of $23 \pm 2$ years were employed as subjects in our experiment. The subjects were made to fill up a consent form and a simple introduction about the research work and stages of the experiment.

\section{B. Visual Cue}

The experiment consists of 3 sessions with 20 trials each conducted on the same day with several minutes break in between. The subjects were asked to move the right and left hand, according to the visual cue displayed on the screen. In each session, the subjects 
were also asked to either move their finger, elbow or shoulder. In each trial, a blank screen was displayed in the first 2 seconds. In the $2^{\text {nd }}$ second a fixation cross ' + ' was displayed on the screen which indicates the beginning of the trial. From the $3^{\text {rd }}$ second onwards, the visual cue (left-right arrow) is displayed. At the same time, the subject was asked to move their respective limb according to the visual cue, until the display is blank again. The timing scheme of the visual cue is given below in Figure 3.

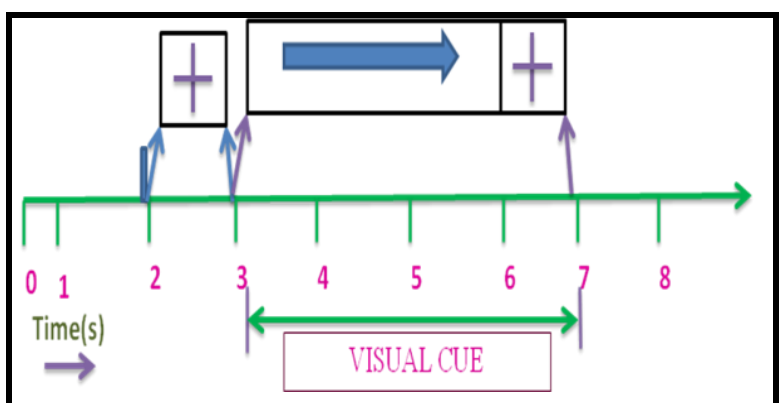

Fig.3. Timing scheme of the experiment

\section{Experimental Setup}

The recording of the EEG signal has been done through NeuroWin, NASAN India with 19 channel $\mathrm{Ag} / \mathrm{AgCl}$ electrodes at a sampling frequency of $250 \mathrm{~Hz}$ and band-pass filtered between $0.01 \mathrm{~Hz}$ and $35 \mathrm{~Hz}$. Only 3 channel electrodes; C3, Cz and C4 were selected and the electrodes are placed according to the International standard 10-20 system, the left ear was selected to be the point for the reference electrode and $\mathrm{FPz}$ as the ground electrode. The sensitivity of the amplifier is set to $100 \mathrm{uV}$ and an additional $50 \mathrm{~Hz}$ notch filter had been utilized to suppress the line noise.

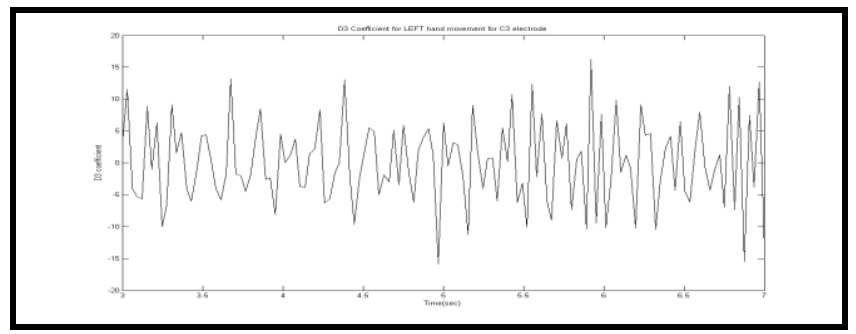

Fig.4a. Left hand movement for C3 electrode

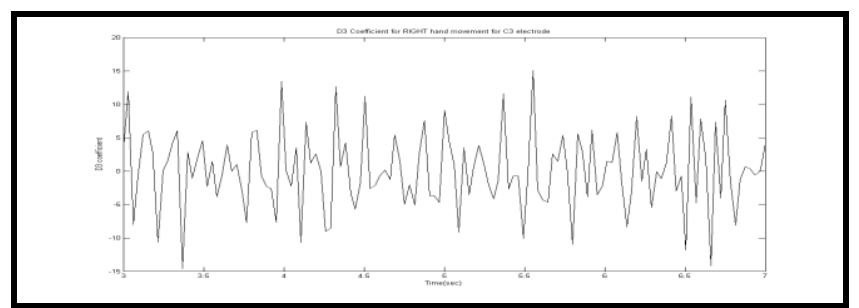

Fig.4c. Right hand movement for C3 electrode

\section{Preprocessing}

For each subject a total of 60 trials were obtained of 8 second each. Out of the three electrodes used, C3 and $\mathrm{C} 4$ are selected for this study as these electrodes have greater relevance for extracting information on the leftright movement. Further, the obtained data was bandpass filtered using an elliptical filter (order 14) between 8 and $30 \mathrm{~Hz}$, for removing the noise based on the environment and recording techniques, and movement related information are mostly obtained in this bandwidth. The training and test data were selected randomly using the 10 fold cross validation technique which would be described later.

\section{E. Wavelet Features Feature Extraction}

In the present study, Daubechies (db) mother wavelet of order 4 is used. After trials with the EEG data, the D3 features and D4 features (Table I) i.e., the difference of the third and fourth level coefficient for the respective electrodes were selected as one of the feature components for the final feature vector (C4-C3). Figure 4 and 5 shows the D3 and D4 wavelet decomposition for left-right imagery for $\mathrm{C} 3$ and $\mathrm{C} 4$ electrode.

TABLE I

EEG SIGNAL DECOMPOSITION INTO FREQUENCY BANDS WITH A SAMPLING FREQUENCY 0F $250 \mathrm{HZ}$

\begin{tabular}{ccc}
\hline $\begin{array}{c}\text { Frequency } \\
\text { Range }\end{array}$ & $\begin{array}{c}\text { Decomposition } \\
\text { Level }\end{array}$ & $\begin{array}{c}\text { Frequency } \\
\text { bands }\end{array}$ \\
\hline $62.5-125$ & D1 & Noise \\
$31.25-62.5$ & D2 & Gamma \\
$15.625-31.25$ & D3 & Beta \\
$7.8125-15.625$ & D4 & Alpha \\
$3.91-7.8125$ & D5 & Delta \\
\hline
\end{tabular}

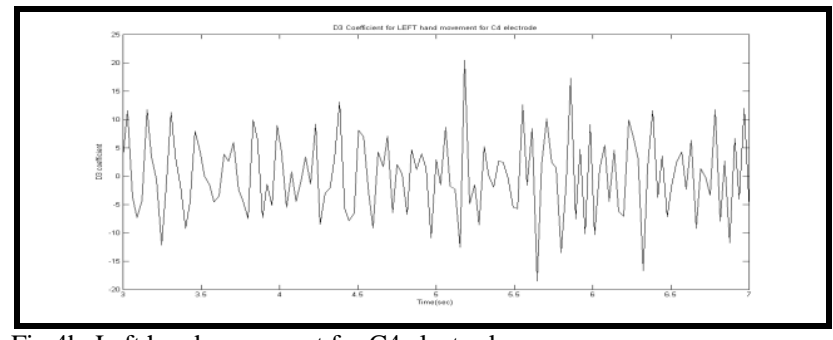

Fig.4b. Left hand movement for C4 electrode

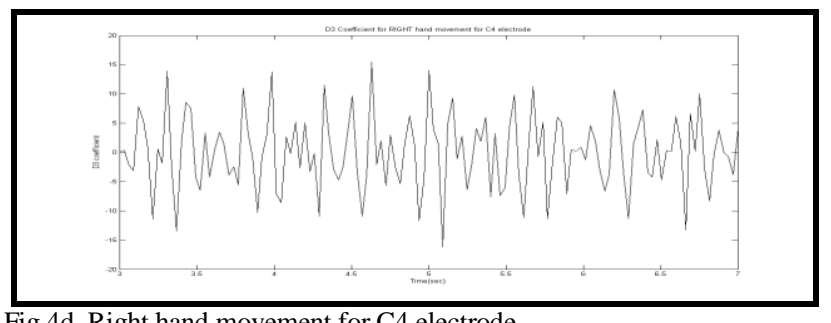

Fig.4d. Right hand movement for C4 electrode

Fig.4. D3 Coefficients for Left/Right movement for C3/C4 electrode 


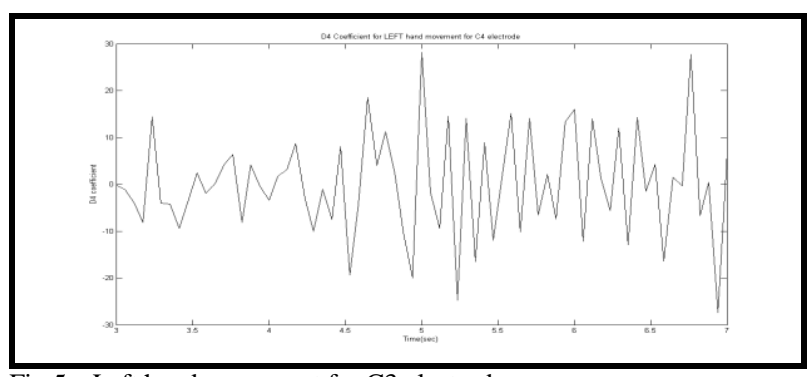

Fig.5a. Left hand movement for C3 electrode

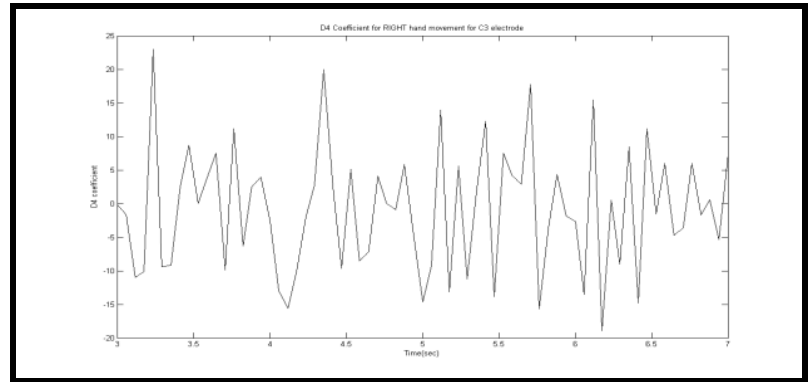

Fig.5c. Right hand movement for $\mathrm{C} 3$ electrode

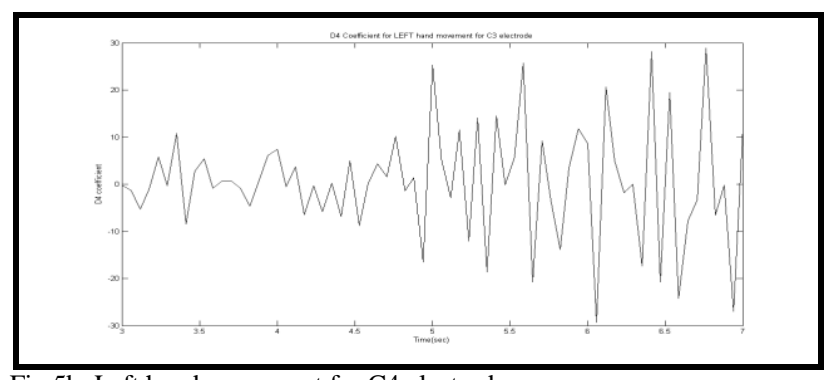

Fig.5b. Left hand movement for C4 electrode

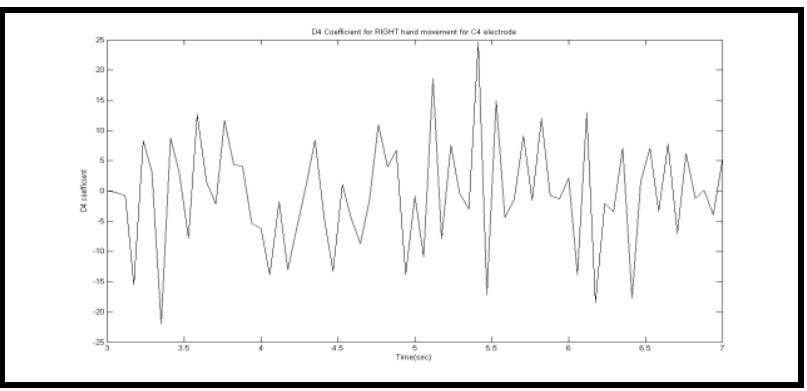

Fig. 5d. Right hand movement for $\mathrm{C} 4$ electrode

Fig.5. D4 Coefficients for Left/Right movement for C3/C4 electrode

\section{F. Spectral Estimation Method}

For this paper, the Welch approach was applied along with a Hamming window of length 125 . The PSD estimates were obtained for the frequency band of 8-25 $\mathrm{Hz}$, which comprises both the alpha or mu band $(8-12 \mathrm{~Hz})$ and the central beta band $(18-25 \mathrm{~Hz})$ for each respective electrode. Then the difference of the PSD estimates (1) and average power (2) is selected as another feature for this study.

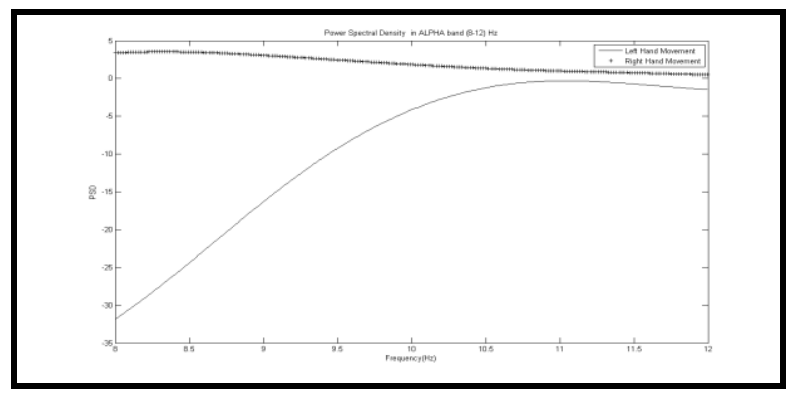

(a)

$$
F_{P S D}=\sum_{f=a}^{b} P S D_{C 4}(f)-\sum_{f=a}^{b} P S D_{C 3}(f)
$$

where $\mathrm{PSD}_{\mathrm{C} 3 / \mathrm{C} 4}$ is the PSD estimates of the respective electrodes in $[a, b]$, where $a \& b$ is the frequency range (8$12 \mathrm{~Hz}$ for alpha band and 18-25 for beta band). Figure 6 shows the power spectral density for left/right hand movement and Figure 7 and 8 shows the power spectral density for elbow/finger/shoulder movement for their respective hand.

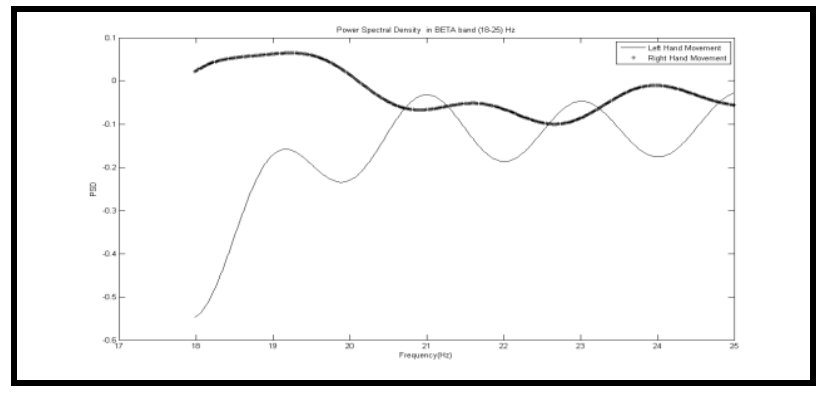

(b)

Figure 6. Power Spectral Density in (a) alpha band (b) beta band for the difference of two electrodes C3 and C4 for left/right hand movement. (- Left Movement; + Right Movement)

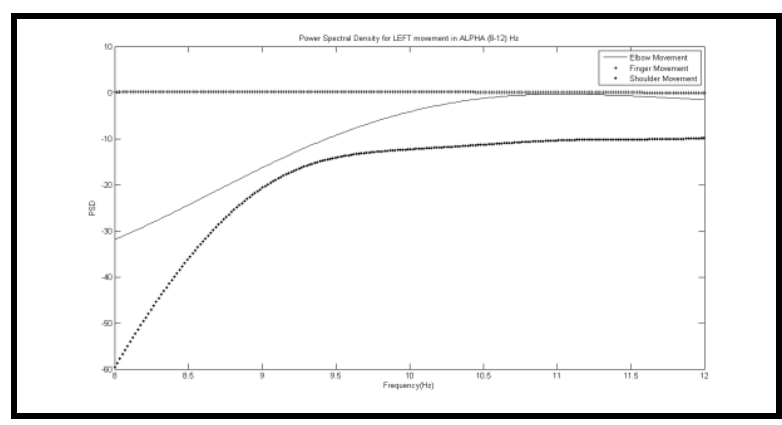

(a)

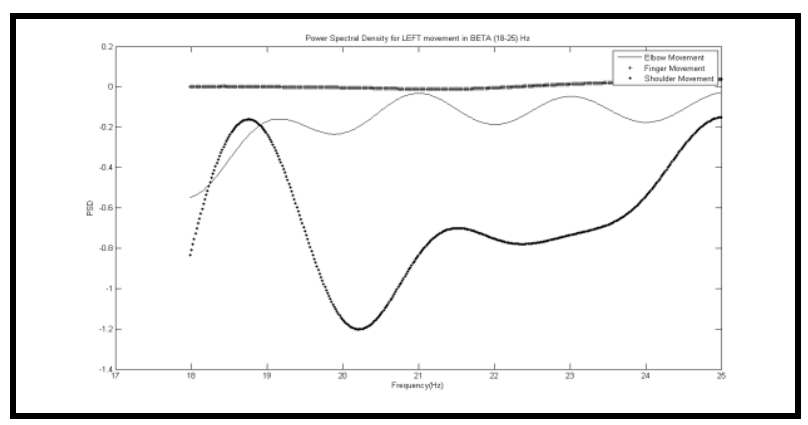

(b)

Figure 7. Power Spectral Density in (a) alpha band (b) beta band for the difference of two electrodes C3 and C4 for elbow/finger/shoulder movement for left hand. ('-' Elbow Movement; '+' Finger Movement; '.' Shoulder) 


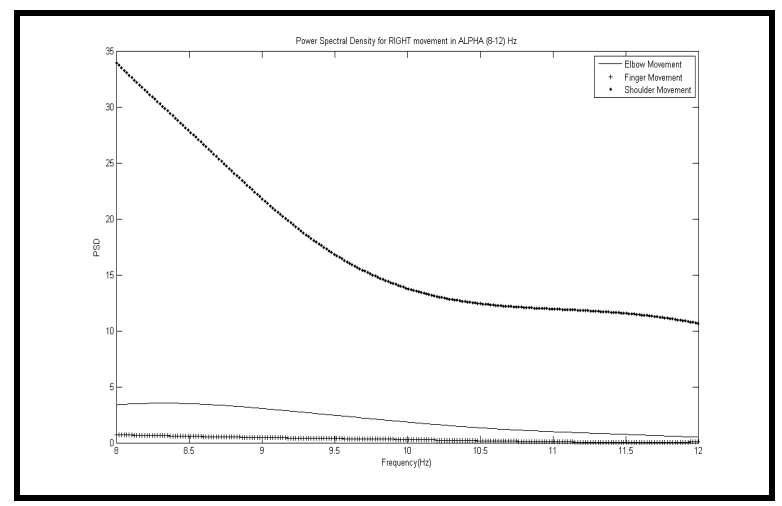

(a)

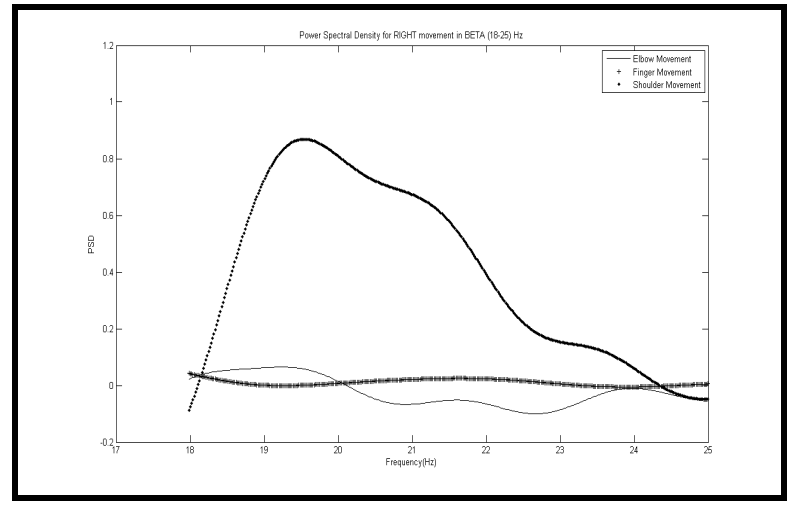

(b)

Figure 8. Power Spectral Density in (a) alpha band (b) beta band for the difference of two electrodes C3 and C4 for elbow/finger/shoulder movement for right hand. ('-'Elbow Movement; '+' Finger Movement; '.' Shoulder)

\section{PERformance AnAlysis}

From the preprocessed filtered signal, only the data from the time interval $\mathrm{t}=3$ to $7 \mathrm{~s}$ are taken, marking the beginning and the end of the visual cue. From the two electrodes of interest, namely, C3 and C4, wavelet coefficients, PSD estimates for the alpha and beta bands and their corresponding powers were selected as the features for this study using the Wavelet toolbox and Signal Processing Toolbox in MATLAB. The classification ability of the obtained feature vectors can be measured through classification accuracy by averaging 10 times over a 10 fold cross validation. The 10 fold cross validation divides the number of samples into 10 disjoint sets, where 9 sets are for training and 1 set for testing. This procedure is repeated 10 times to obtain an average classification result. For our study, we have employed a two fold classification; i) left-right movement classification, and, ii) finger-elbow-shoulder movement classification. The average classification results are given in for 8 subjects using QDA and SVM (Linear and RBFKernel) based approaches. Since SVM is a binary class classifier, the one-against-all approach is employed for the elbow-finger-shoulder classification, i.e., for example, this approach pits the elbow movement with the rest of the movement (finger-shoulder movement) and the same for the rest of the movements. Table II and III gives the classification results for left/right hand movement for their respective features, and Table IV and $\mathrm{V}$ gives the classification results classification results for left/right hand movement for their respective features, and Table IV and $\mathrm{V}$ gives the for elbow/finger/shoulder movement for the same. For left-right hand movement, the maximum classification accuracy of $87.50 \%$ is obtained using wavelet coefficient for RSVM classifier. Also by using PSD as the feature set, the maximum classification accuracy of $87.35 \%$ is obtained for RSVM classifier.

For the multi-class classification, i.e., Finger-ElbowShoulder classification the maximum classification accuracy of $80.11 \%$ for elbow, $93.26 \%$ for finger and $81.12 \%$ for shoulder is obtained using the features obtained from power spectral density for RSVM classifier. While by taking the wavelet coefficient as feature set, the maximum classification accuracy of $74.24 \%$ for RSVM classifier, $72.43 \%$ for LSVM classifier and $72.12 \%$ for RSVM classifier for elbow, finger and shoulder, respectively. In turn QDA gives an average classification accuracy, as shown in Table II, III, IV and V. All the programming was done in "offline" mode using MATLAB environment.

TABLE II

RESULT OF LEFT/RIGHT CLASSIFICATION FOR WAVELET FEATURES

\begin{tabular}{|c|c|c|c|c|}
\hline \multirow[t]{2}{*}{ Subject ID } & \multirow[t]{2}{*}{ Features } & \multicolumn{3}{|c|}{ Classification Accuracy } \\
\hline & & QDA & LSVM & RSVM \\
\hline 1 & & $.7942+/ .0541$ & $.8333+/-.1700$ & $.8615+1-.0621$ \\
\hline 2 & & $.6667+1-.0786$ & $.6092+/ .0959$ & $.7610+1-.0539$ \\
\hline 3 & & $.7667+/-.1956$ & $.7500+/-.0627$ & $.8697+1-.0593$ \\
\hline 4 & Wavelet (D3 & $.5556+1-.0962$ & $.4078+/-.1138$ & $.5478+1-.0315$ \\
\hline 5 & \& D4 Coeff.) & $.5000+/ .2079$ & $.6457+/ .0589$ & $.6392+1-.0852$ \\
\hline 6 & & $.8326+/-.0222$ & $.6449+/-.0625$ & $.8750+1-.0546$ \\
\hline 7 & & $.7500+/-.1179$ & $.5721+/-.0607$ & $.7623+1-.0438$ \\
\hline 8 & & $.7333+/-.1956$ & $.6204+/-.0456$ & $.7352+/-.0500$ \\
\hline
\end{tabular}

TABLE III

RESULT OF LEFT/RIGHT CLASSIFICATION FOR PSD FEATURES

\begin{tabular}{|c|c|c|c|c|}
\hline \multirow[t]{2}{*}{ Subject } & \multirow[t]{2}{*}{ Features } & \multicolumn{3}{|c|}{ Classification Accuracy } \\
\hline & & QDA & LSVM & RSVM \\
\hline 1 & \multirow{8}{*}{$\begin{array}{l}\text { PSD (Alpha } \\
\text { \& Beta Band) }\end{array}$} & $.7717+/-.0567$ & $.7774+1-.0480$ & $.7744+1-.0389$ \\
\hline 2 & & $.5075+1-.0261$ & $.7749+1-.0457$ & $.8503+1-.0671$ \\
\hline 3 & & $.7535+/-.0516$ & $.7594+/-1006$ & $.8024+1-.0747$ \\
\hline 4 & & $.5909+/-0511$ & $.6178+/-.1512$ & $.7147+/-.1170$ \\
\hline 5 & & $.6348+/-.1255$ & $.6851+/-.0735$ & $.6411+1-.0490$ \\
\hline 6 & & $.7745+/ .0249$ & $.8616+/ \cdot .0446$ & $.8735+/-.0853$ \\
\hline 7 & & $.6176+/-.0732$ & $.7305+1-.0366$ & $.7516+1-.0543$ \\
\hline 8 & & $.7578+/-.0393$ & $.6071+/-.0444$ & $.8047+1-.0302$ \\
\hline
\end{tabular}


TABLE IV

RESULT OF ELBOW/FINGER/SHOULDER CLASSIFICATION FOR WAVELET FEATURES

\begin{tabular}{|c|c|c|c|c|c|c|c|c|}
\hline \multirow[t]{2}{*}{ Subject } & \multirow[t]{2}{*}{ Features } & \multicolumn{7}{|c|}{ Classification Accuracy } \\
\hline & & QDA & LSVM-E & LSVM-F & LSVM-S & RSVM-E & RSVM-F & RSVM-S \\
\hline 1 & \multirow{8}{*}{$\begin{array}{c}\text { Wavelet ( } \\
\text { D3 \& D4 } \\
\text { Coeff.) }\end{array}$} & $.6527+/-.0435$ & $.5847+/-.0484$ & $.6112+/-.0302$ & $.6166+/-.0736$ & $.6508+/-.0503$ & $.6368+/-.0548$ & $.5753+/-.0990$ \\
\hline 2 & & $.4083+/-.2306$ & $.6714+/-.0485$ & $.4648+/-.0752$ & $.4645+/-.0425$ & $.6619+/-.0596$ & $.6383+/-.0269$ & $.6999+/-.0555$ \\
\hline 3 & & $.7017+/-.1922$ & $.6002+/-.0455$ & $.5284+/-.0606$ & $.5454+/-.0865$ & $.7424+/-.0718$ & $.5025+/-.0668$ & $.6123+/-.0603$ \\
\hline 4 & & $.4444+/-3469$ & $.5863+/-.0465$ & $.5793+/-.0381$ & $.4384+/-.0384$ & $.7301+/-.0702$ & $.6329+/-.0539$ & $.6370+/-.0948$ \\
\hline 5 & & $.3833+/-.2086$ & $.4678+/-.0396$ & $.594+/-.0676$ & $.5079+/-.0656$ & $.6864+/-.0272$ & $.5923+/-.0884$ & $.7212+/-.0914$ \\
\hline 6 & & $.6080+/-.1041$ & $.5298+/-.0315$ & $.7243+/-.0282$ & $.7086+/-.0551$ & $.6044+/-.0985$ & $.6936+/-.1138$ & $.6833+/-.0157$ \\
\hline 7 & & $.7300+/-.2236$ & $.6937+/-.1166$ & $.5773+/-.0543$ & $.6229+/-.0512$ & $.6891+/-.0628$ & $.5968+/-.0363$ & $.6704+/-.0344$ \\
\hline 8 & & $.6900+/-.2049$ & $.5157+/-.0678$ & $.6823+/-.0328$ & $.5420+/-.0295$ & $.6863+/-.0430$ & $.6137+/-.0529$ & $.6272+/-.0633$ \\
\hline
\end{tabular}

-e'-elbow classification; '-f' -finger classification; '-s'- shoulder classification

TABLE V

RESULT OF ELBOW/FINGER/SHOULDER CLASSIFICATION FOR PSD FEATURES

\begin{tabular}{ccccccccc}
\hline Subject & Features & \multicolumn{5}{c}{ Classification Accuracy } \\
\hline & & QDA & LSVM-E & LSVM-F & LSVM-S & RSVM-E & RSVM-F & RSVM-s \\
\hline $\mathbf{1}$ & & $.5919+/-.0603$ & $.6505+/-.0268$ & $.6820+/-.0741$ & $.6470+/-.0573$ & $.6237+/-.0568$ & $.6701+/-.0626$ & $.6913+/-.0582$ \\
$\mathbf{2}$ & & $.4644+/-.0652$ & $\mathbf{. 8 5 0 9 + / - . 0 9 6 3}$ & $\mathbf{. 9 0 9 3 + / - . 0 7 8 0}$ & $.6807+/-.0330$ & $.7574+/-.0490$ & $\mathbf{. 9 3 2 6 + / - . 0 3 0 7}$ & $\mathbf{. 8 1 1 2 + / - . 0 6 7 9}$ \\
$\mathbf{3}$ & & $\mathbf{. 6 3 5 5 + / - . 0 8 5 9}$ & $.6868+/-.0348$ & $.6311+/-.0886$ & $.6101+/-.0498$ & $.6560+/-.0747$ & $.5682+/-.0983$ & $.6786+/-.0713$ \\
$\mathbf{4}$ & PSD (Alpha & $.3972+/-.0655$ & $.6026+/-.0968$ & $.5808+/-.1542$ & $.5564+/-.0495$ & $.5869+/-.0781$ & $.6653+/-.0339$ & $.6980+/-.0639$ \\
$\mathbf{5}$ & $\mathbf{\&}$ Beta band) & $.4064+/-.0897$ & $.6335+/-.0371$ & $.5942+/-.0459$ & $.5269+/-.1264$ & $.6696+/-.0371$ & $.6212+/-.0491$ & $.7092+/-.0468$ \\
$\mathbf{6}$ & & $.6173+/-.0361$ & $.5160+/-.0676$ & $.6325+/-.0542$ & $.6549+/-.0402$ & $\mathbf{. 8 0 1 1 + / - . 0 6 5 8}$ & $.5623+/-.0975$ & $.7000+/-.0650$ \\
$\mathbf{7}$ & & $.4767+/-.0638$ & $.6546+/-.0678$ & $.6785+/-.0395$ & $.6225+/-.0819$ & $.6566+/-.0314$ & $.5864+/-.0919$ & $.6736+/-.0316$ \\
$\mathbf{8}$ & & $.5560+/-.0563$ & $.6067+/-.0927$ & $.6828+/-.0616$ & $\mathbf{. 6 9 3 2 + / - . 0 6 4 5}$ & $.6429+/-.0722$ & $.6256+/-.0626$ & $.6477+/-.0804$ \\
\hline
\end{tabular}

-e'-elbow classification; '-f' -finger classification; '-s'- shoulder classification

\section{CONCLUSION}

This work addresses the ability to differentiate the EEG signal to its corresponding left-right movement and elbow-finger-shoulder movement. Wavelet transform and power spectral density estimate are techniques followed in this study for feature extraction. The results presented in this paper indicates that elbow-finger-shoulder movement can be successfully classified using the given set of features, and further this opens up newer avenue for classifications based on composite decision. Experimental results showed that kernelized SVM (RBF-based) showed a superior classification result for multi-class classification.

In light of our present findings, our approach for future work involves the improvement of the accuracy of the classifiers. Also the combination of feature vector is a vital step for proper classification, thus newer features are needed to be tried out which would be simple, robust and require less computational time with higher accuracy, which would be more apt to control EEG based BCI devices. Future study in this direction will aim at techniques for optimizing feature selection, extraction and classification methodologies to be implemented in online classification of EEG data for BCI research.

\section{REFERENCES}

[1] Nijholt Anton, Tan Desney, "Brain computer interfacing for intelligent systems," IEEE intelligent systems, 2008

[2] McFarland Dennis J., Wolpaw Jonathan R. "Brain-Computer Interface Operation of Robotic and Prosthetic Devices", IEEE Computer Society, 2008

[3] Daly Janis J, Wolpaw Jonathan R , "Brain-computer interfaces in neurological rehabilitation", Lancet Neurol 2008; 7: 1032-43

[4] Schwartz A.B., Cui X.T., Weber D.J., Moran D.W. "Brain Controlled Interfaces: Movement Restoration using Neural Prosthetics." Neuron 52, October 2006, 205-220

[5] Lebedev M.A., Nicoleis, "Brain-machine interface: Past, present and future", Trends Neurosci. 29(9), September 2006, 536-546

[6] Vaughan T.M., Heetderks W.J., Trejo L.J., Rymer W.Z., Weinrich M., Moore M.M., Kubler A., Dobkin B.H., Birbaumer N., Donchin E., Wolpaw E.W., Wolpaw J.R. " Brain computer interface technology: A review of the second international meeting", IEEE Trans. Neural Syst. Rehab. Eng. 11(2), June 2003, 94-109

[7] Wolpaw J.R., Birbaumer N., Heetderks W.J., McFarland D.J., Peckham P.H., Schalk G., Donchin E., Quatrano L.A., Robinson C.J., Vaughan T.M. " Brain computer interface : A review of the first international meeting”, IEEE Trans. Rehabilitation Eng. 8(2), June 2000, 164-173

[8] Pfurtscheller G., Neuper C., "Motor imagery activates primary sensorimotor area in humans", Neuroscience Letters 239 , December 1997, 65-68

[9] Pilla Jr, V. Lopes H.S.,"Detection of movement related Desynchronization of the EEG using neural networks", Proceedings of the $22^{\text {nd }}$ Annual EMBS International Conference, Chicago, July 2000

[10] Qiang C., Hu P., Huanqing F., “ Experiment study of the relation between motion complexity and event related Desynchronization/synchronization”, 2005 First International 
Conference on Neural interfaces and Control Proceedings, China, May 2005

[11] Matsunanga T., Katayama Y., Hayami T., Iramina K. "Measurement of mu/beta ERD and gamma ERS during the imagination of body parts movement." $30^{\text {th }}$ Annual International IEEE EMBS Conference Vancouver Canada, August 2008

[12] Hema C.R., Paulraj M.P., Yaacob S., Adom A.H., Nagarajan R. "Recognition of motor imagery of hand movements for a BMI using PCA features." 2008 International Conference on Electronic Design, Penang, Malaysia, December 2008

[13] Thomas K.P., Guan C., Tong L.C., Prasad V.A. "An adaptive filter bank for motor imagery based brain computer interface." $30^{\text {th }}$ Annual International IEEE EMBS Conference, Vancouver, Canada, August 2008

[14] Ince N.F., Goksu F., Tewfik A.H., Arica S., "Adapting subject specific motor imagery EEG patterns in space-time-frequency for a brain computer interface." Biomedical signal processing and control 4, 2009, 236-246

[15] Pfurscheller G., Neuper C., Schlogl A., Lugger K. "Separability of EEG signals recorded during right and left motor imagery using Adaptive Autoregressive Parameters." IEEE transaction on rehabilitation engineering 6 (3), September 1998, 316-325

[16] Cososchi S., Strungaru R., Ungureanu A., Ungureanu M., " EEG feature extraction for motor imagery", Proc. of IEEE Engineering in Medicine and Biology Society, August 2006, 1142-1146

[17] Yom-Tov E., Inbar G.F., "Feature selection for the classification of movements from single movement-related potentials", IEEE Trans. Neural Syst. Rehab. Eng. 10, 2002, 170-178

[18] Zhendong Mu Dan Xiao, Jianfeng Hu. "Classification of motor imagery EEG signals based on STFTs"

[19] Schloegl A., "Dynamic spectral analysis based on an autoregressive model with time-varying coefficients", IEEEEMBC and CMBEC, 1995

[20] Vidaurre C., Kramer N., Blankertz B., Schlogl A. "Time Domain Parameters as a feature for EEG-based Brain Computer Interface." Neural Networks 22, 2009, 1313-1319

[21] Mahmoudi B., Erfanian A. "Single channel EEG based prosthetic hand grasp control for amputee subjects." Proceedings of the Second Joint EMBS/BMES Conference, Houston, Texas, USA, October 2002

[22] Darvishi S., Al-Ani A. "Brain-computer interface analysis using continuous wavelet transform and adaptive neuro-fuzzy classifier", Proc. $29^{\text {th }}$ Int. Annu. Conf. IEEE Eng. Med. Biol. Soc., August 2007, 3220-3223

[23] Huang R. S., Kuo C. J., Tsai L.-L., and Chen O. T. C., "EEG pattern recognition-arousal states detection and classification," Proc. IEEE Int. Conf. Neural Netw. 2, June 1996, 641-646.

[24] Rezaei S., Tavakolian K., Nasrabadi A. M., and Setarehdan, S. K. "Different classification techniques considering brain computer interface applications," J. Neural. Eng. 3(2), Jun 2006, 139-144

[25] Xu W., Guan C., Siong C. E., Ranganatha S., Thulasidas M., Wu J., "High accuracy classification of EEG signal," Proc. 17th Int. Conf. Patt. Recog. ICPR 2, 2004, 391-394.

[26] Thulasidas M., Guan C., and Wu J., "Robust classification of EEG signal for brain computer interface," IEEE Trans. Neural Syst. Rehab. Eng. 14(1), Mar 2006, 24-29

[27] Flotzinger D., Pfurtscheller G., Neuper C., Mohl W, Berger H, "Classification of non-averaged EEG data by learning vector quantization and the impact of signal processing"

[28] Pregeiizer M., Pfurtschcller G., "Distinction Sensitive Learning Vector Quantization (Dslvq) - Application as a Classifier based Feature Selection Method for a Brain Computer Interface"

[29] Bhattacharyya S., Khasnobish A., Konar A., Tibarewala D.N., "Performance analyisis of LDA, QDA and KNN algorithms in leftright limb movement classification from EEG data", Accepted for oral presentation in International conference on systems in medicine and biology, IIT Kharagpur, 2010

[30] Khasnobish A., Bhattacharyya S., Konar A., Tibarewala D.N., "KNearest neighbor classification of left-right limb movement using EEG data.", Accepted for oral presentation in International conference on Biomedical Engineering and assistive technologies, NIT Jalandhar, 2010.

[31] Sanei S., Chambers J.A., "EEG Signal processing", $1^{\text {st }}$ ed., John Wiley \& Sons Ltd., 2007
[32] Xu Q., Zhou H., Wang Y., Huang J. "Fuzzy support vector machine for classification of EEG signals using wavelet based features." Medical Engineering \& Physics 31, 2009, pp. 858-865

[33] Herman P., Prasad G., McGinnity T.M., Coyle D. "Comparative analysis of spectral approaches to feature extraction for EEG-based motor imagery classification.” IEEE Trans. Neural sys. Rehab eng. 16(4), August 2008, pp. 317-326

[34] D.G. Childers, ed., Modern Spectrum Analysis, New York: IEEE Press, 1978

[35] Gunn Steve R.,"Support Vector Machines For Classification and Regression", Technical report, University of Southampton, May 1998 .

[36] BURGES CHRISTOPHER J.C., "A Tutorial on Support Vector Machines for Pattern Recognition", Kluwer Academic Publishers, Boston, pp- 1-43

[37] V. Vapnik. "The Nature of Statistical Learning Theory". SpringerVerlag, New York, NY, 1995.

[38] Srivastava Santosh, Gupta Maya R., Frigyik B'ela A., "Bayesian Quadratic Discriminant Analysis" Journal of Machine Learning Research 8 (2007) 1277-1305

[39] J. Ye., "Characterization of a family of algorithms for generalized discriminant analysis of undersampled problems." Journal of Machine Learning Research, 6:483-502, 2005. 